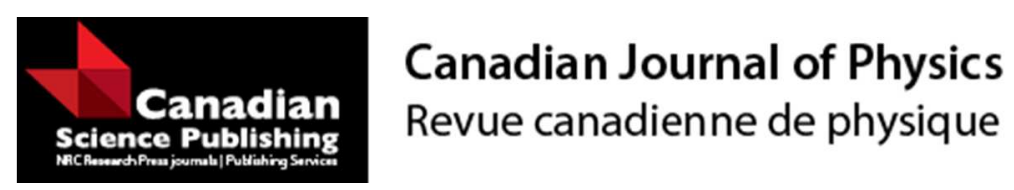

\title{
Phase-shifts determination for nucleon-nucleon scattering using velocity-dependent potentials
}

\begin{tabular}{|r|l|}
\hline Journal: & Canadian Journal of Physics \\
\hline Manuscript ID & cjp-2015-0600.R1 \\
\hline Manuscript Type: & Article \\
\hline Date Submitted by the Author: & 23-Oct-2015 \\
\hline Complete List of Authors: & Sayyed, M.I.; University of Tabuk \\
\hline Keyword: & $\begin{array}{l}\text { scattering phase shifts, velocity-dependent potential, finite square well, } \\
\text { Yukawa potential., Schrödinger equation }\end{array}$ \\
\hline \multicolumn{2}{|c}{} \\
\hline
\end{tabular}

SCHOLARONE $^{\text {th }}$
Manuscripts 


\title{
Phase-shifts determination for nucleon-nucleon scattering using velocity-dependent potentials
}

\author{
M. I. Sayyed* \\ Department of physics, University of Tabuk, Tabuk, Kingdom of Saudi Arabia
}

\begin{abstract}
The S-wave time-independent Schrödinger equation with an isotropic velocity-dependent potential is considered.We have used perturbation theory to calculate the scattering phase shifts when the energy is changed by a small amount $\Delta \mathrm{E}$ from an arbitrary unperturbed value $\mathrm{E}_{0}$. The validity of our results were tested by comparing the perturbed phase shifts to those obtained exactly by solving Schrödinger equation. We assumed the local potential to have the form of a finite square well and the velocity dependent part of the potential to have the form of a Yukawa potential.
\end{abstract}

Keywords: scattering phase shifts; velocity-dependent potential; finite square well; Yukawa potential.

\section{Introduction}

The energy dependence of the scattering phase shifts as we move away from the zero energy limit has been the interest of many old and recent works. In nuclear physics field a lot of what we know about nuclei and elementary particles comes from analyzing the nucleon-nucleon scattering data at low energies. For example; Romo and Valluri [1] studied the momentum dependence of the scattering phase shift for potentials that consist of the sum of a screened Coulomb potential and a short range potential. In addition Milward and Wilkin [2] derived analytical expressions for the changes in the scattering wave functions and the corresponding changes in the phase shifts by introducing a small change in a local potential. However, they performed their calculations starting from the probability density equation rather than the Schrödinger equation. The changes in the wave functions and phase shifts were shown to depend on the unperturbed wave function and the perturbing potential.

* e-mail: mabualssayed@ut.edu.sa 
In addition, Blatt and Jackson [3] applied variational methods developed by Schwinger to neutron-proton scattering in the energy limit less than $10 \mathrm{MeV}$. By considering the s-wave scattering alone and ignoring the tensor force the authors obtained an expansion for the phase shift in powers of energy, which is known as the effective range expansion.

In this work the Schrödinger equation with a velocity-dependent potential will be considered. Our aim is to investigate the energy dependence of the scattering phase shifts and the corresponding wave functions when the incident energy is perturbed by a small amount $\Delta \mathrm{E}$ from its original value $\mathrm{E}_{0}$.

Recently velocity-dependent potentials have been employed in the nucleon-nucleon scattering to replace the hard core in nucleon-nucleon interactions. There have been many studies and applications [ 4-13 ] of the velocity-dependent potentials. The velocity-dependent potential consists of two parts; a local potential $\mathrm{V}(\mathrm{r})$ and a velocity-dependent $\tilde{V}(r, p)$ potential, as follows $[14,15]$

$$
\begin{aligned}
\widehat{V}(r, p) & =V(r)+\tilde{V}(r, p) \\
& =V(r)+\frac{\hbar^{2}}{2 m}\left\{\rho(r) \nabla^{2}+\nabla \rho(r) . \nabla\right\}
\end{aligned}
$$

where $\rho(r)$ is the isotropic form factor function of the radial variable $r$ assumed to have a common range $\mathrm{b}$ beyond which it identically vanishes.

Generally, $\rho(r)$ can be taken to represent the spatially varying nuclear density. The first term on the right is essentially a kinetic energy term and hence the name velocity-dependent potential, which combines with the kinetic-energy term in the Schrödinger equation. However, the second term is proportional to the rate of change of density and hence it is sensitive to the diffuse edge of nuclei.

In terms of reduced wave function $u(r)=r R(r)$ the radial Schrödinger equation for a particle of mass $m$ and energy $E$ moving under the isotropic, velocity-dependent potential may be written by 


$$
(1-\rho(r)) u^{\prime \prime}(\mathrm{r})-\left[u^{\prime}(\mathrm{r})-\frac{u(r)}{r}\right] \rho^{\prime}(r)=\frac{2 m}{\hbar^{2}}[V(r)-E] u(r),
$$

where the prime denotes differentiation with respect to the radial variable $r$.

In section 2 we shall investigate the energy dependence of the wave functions and phase shift when the energy of the incident particle is changed from its unperturbed value $\mathrm{E}_{0}$ by a small amount $\Delta \mathrm{E}$. We expand $k \cot \delta$, where $\delta$ is the perturbed phase shift as a power series in $\Delta \mathrm{E}$ and obtained analytical formulas for the effective range expansion parameters in the perturbing energy. In section3 we shall consider an explicit example to calculate the perturbed phase shifts using the perturbation approach, we assumed the local potential to have the form of finite square well but the velocity dependent part of the potential to have the form of Yukawa potential. In this example we cannot find exact analytical solutions to Schrödinger equation, hence we have solved Schrödinger equation numerically to determine the exact phase shifts. Finally, the conclusions are presented in section 4.

\section{Energy dependence of the wavefunction and phase shift}

We shall deal with the S-wave scattering from a potential and we assumed that the local and velocity dependent parts of the potential to have a given radius $b$ beyond which both identically vanish. It is customary when deriving the effective range expansion to choose a normalization such that, for distances larger than $b$ the wave function behaves like [16]

$$
u(k, r)=\frac{\sin (k r+\delta)}{\sin \delta}
$$

where $\delta$ is the exact $\mathrm{S}$-wave phase shift and $E=\hbar^{2} \mathrm{k}^{2} / 2 \mathrm{~m}$ is the incident energy.

Expand the $\mathrm{S}$-wave function as a power series in $\mathrm{k}^{2}$ about some value $\mathrm{k}_{0}^{2}$;

$$
u(k, r)=\sum_{n=0}^{\infty}\left(\frac{\hbar^{2}}{2 m}\right)^{n} \frac{\left(k^{2}-k_{\mathrm{o}}^{2}\right)^{n}}{n !} u_{n}\left(k_{\mathrm{o}}, r\right)
$$

Similarly

$$
\alpha(k)=k \cot \delta=\sum_{\mathrm{n}} \frac{1}{\mathrm{n} !}(\Delta E)^{n} \alpha_{n}=\sum_{\mathrm{n}} \frac{1}{\mathrm{n} !}\left(\frac{\hbar^{2}}{2 \mathrm{~m}}\right)^{\mathrm{n}}\left(k^{2}-k_{\mathrm{o}}^{2}\right)^{\mathrm{n}} \alpha_{n}
$$


where $\delta$ is the perturbed phase shift. The conventional scattering length and effective range are given by $\mathrm{a}_{0}=-1 / \alpha_{0}, \mathrm{r}_{0}=2 \alpha_{1}$ respectively.

Clearly, the larger-dependence of the first order change in $u(k, r)$ is fixed by differentiating (4) with respect to $k^{2}$ then setting $k=k_{\mathrm{o}}$. Using the expression for $u(k, r)$ given in (3) we obtain:

$$
u_{1}\left(k_{\mathrm{o}}, r\right)=\frac{2 m}{\hbar^{2}}\left(\frac{r \cos \left(k_{\mathrm{o}} r+\delta_{\mathrm{o}}\right)}{2 k_{\mathrm{o}} \sin \delta_{\mathrm{o}}}-\frac{\delta^{\prime} \sin k_{\mathrm{o}} r}{2 k_{\mathrm{o}} \sin ^{2} \delta_{\mathrm{o}}}\right)
$$

Where $\delta^{\prime}=\frac{d \delta}{d k}$ is evaluated at $\left(k=k_{o}\right)$ and the subscript (o) denotes the unperturbed case i. e, $\Delta \mathrm{E}=0$

\subsection{First order wavefunction correction}

In order to derive the first-order wavefunction correction when the incident energy is perturbed by a small amount $\Delta \mathrm{E}$ from the initial value $\mathrm{E}_{0}$, we have started by inserting (4) into (2), we find that the coefficient of $(\Delta E)^{n}$, where $n \geq 1$ satisfies the following equation [17]

$$
\begin{gathered}
(1-\rho(r)) \frac{u_{\mathrm{n}}^{\prime \prime}(r)}{\mathrm{n} !}-\left(\frac{u_{\mathrm{n}}^{\prime}(r)}{\mathrm{n} !}-\frac{u_{\mathrm{n}}(r)}{\mathrm{n} ! r}\right) \rho^{\prime}(r) \\
=\frac{2 \mathrm{~m}}{\hbar^{2}}\left(\mathrm{~V}(r)-E_{\mathrm{o}}\right) \frac{u_{\mathrm{n}}(r)}{\mathrm{n} !} \\
-\frac{2 \mathrm{~m}}{\hbar^{2}} \frac{u_{\mathrm{n}-1}(r)}{(\mathrm{n}-1) !} .
\end{gathered}
$$

Set $\Delta \mathrm{E}=0$ (i.e. The perturbation is switched off) into the last equation we get the unperturbed Schrödinger equation;

$$
(1-\rho(r)) u_{\mathrm{o}}^{\prime \prime}(r)-\left(u_{\mathrm{o}}^{\prime}(r)-\frac{u_{\mathrm{o}}(r)}{r}\right) \rho^{\prime}(r)=\frac{2 m}{\hbar^{2}}\left(\mathrm{~V}(r)-E_{\mathrm{o}}\right) u_{\mathrm{o}}(r),
$$

where $\mathrm{u}_{0}(\mathrm{r})$ is the unperturbed wavefunction.

To find the first-order wavefunction correction we set $n=1$ in (7) and evaluating for the term 
$2 \mathrm{~m} / \hbar^{2}\left(\mathrm{~V}(\mathrm{r})-\mathrm{E}_{0}\right)$ using the unperturbed equation (8) leads to

$$
\begin{gathered}
(1-\rho(r)) u_{1}^{\prime \prime}(r)-\left[u_{1}^{\prime}(r)-\frac{u_{1}(r)}{r}\right] \rho^{\prime}(r)=-\frac{2 m}{\hbar^{2}} u_{o}(r) \\
+u_{1}(r)(1-\rho(r)) \frac{u_{o}^{\prime \prime}(r)}{u_{o}(r)}-\left[u_{o}^{\prime}(r)-\frac{u_{o}(r)}{r}\right] \frac{\rho^{\prime}(r) u_{1}(r)}{u_{o}(r)}
\end{gathered}
$$

After multiplying both sides by $\mathrm{u}_{0}(\mathrm{r})$ and rearranging results in

$$
\begin{gathered}
(1-\rho(r))\left[u_{o}(r) u_{1}^{\prime \prime}(r)-u_{1}(r) u_{o}^{\prime \prime}(r)\right]+\left[u_{o}^{\prime}(r) u_{1}(r)-u_{o}(r) u_{1}^{\prime}(r)\right] \rho^{\prime}(r) \\
=-\frac{2 m}{\hbar^{2}} u_{o}^{2}(r)
\end{gathered}
$$

The left hand side is an exact derivative hence, we may write

$$
\frac{d}{d r}\left[(1-\rho(r))\left(u_{1}^{\prime}(r) u_{o}(r)-u_{1}(r) u_{o}^{\prime}(r)\right)\right]=-\frac{2 m}{\hbar^{2}} u_{o}^{2}(r)
$$

Integrating the last equation from the origin to $r$ and using the condition $u_{n}(0)=0$ for $\mathrm{n} \geq 0$ leads to:

$$
\frac{d}{d r}\left(\frac{u_{1}(r)}{u_{o}(r)}\right)=-\frac{2 m}{\hbar^{2}} \frac{1}{(1-\rho(r))} \int_{0}^{r} u_{o}^{2}(r) d r
$$

A second integration from $r=b$ to $r$ we get the first-order wavefunction namely,

$$
\begin{aligned}
u_{1}(r)=-\frac{2 m}{\hbar^{2}} & u_{o}(r) \int_{b}^{r} \frac{d s}{(1-\rho(s)) u_{o}^{2}(s)} \int_{0}^{t} u_{o}^{2}(t) d t \\
& +C_{1} u_{o}(r)
\end{aligned}
$$

where $\mathrm{C}_{1}$ is the constant of integration given by $C_{1}=u_{1}(b) / u_{0}(b)$. 


\subsection{Energy dependence of the phase shift}

In order to study the energy dependence of the phase shift when the incident energy is changed from its unperturbed value $\mathrm{E}_{0}$, we start from (5), clearly the $\alpha_{n}$ terms of the series are given by

$$
\alpha_{\mathrm{n}}=\left.\left(\frac{2 m}{\hbar^{2}}\right)^{n}\left(\frac{\partial}{\partial k^{2}}\right)^{n} k \cot \delta\right|_{k=k_{\mathrm{o}}}
$$

Setting $n=1$ in the above equation results in the following expressions for $\alpha_{1}$,

$$
\alpha_{1}=\frac{2 m}{\hbar^{2}}\left[\frac{\cot \delta_{\mathrm{o}}}{2 k_{\mathrm{o}}}-\frac{\delta_{0}^{\prime}}{2 \sin ^{2} \delta_{\mathrm{o}}}\right]
$$

In order to find $\alpha_{1}$ we need to determine $\delta_{0}{ }^{\prime}$, to achieve this we shall start by considering (2) for two energies $E_{1}=\frac{\hbar^{2} k_{1}^{2}}{2 m}$ and $E_{2}=\frac{\hbar^{2} k_{2}^{2}}{2 m}$ [15]. Consequently use (2) we may write

$$
\left.\left[u_{2}(r) u_{1}^{\prime}(r)-u_{1}(r) u_{2}^{\prime}(r)\right]\right|_{r=b}+\int_{0}^{b}\left(k_{1}^{2}-k_{2}^{2}\right) u_{2}(r) u_{1}(r) d r=0
$$

Differentiate (16) with respect to $k_{2}$ and then letting $k_{2} \rightarrow k_{1}=k_{0}$ we get

$$
\left.\frac{1}{2 k_{0}}\left[u_{o}^{\prime}(r) \frac{\partial u_{o}(r)}{\partial k_{\mathrm{o}}}-u_{o}(r) \frac{\partial u_{o}^{\prime}(r)}{\partial k_{\mathrm{o}}}\right]\right|_{r=b}=\int_{0}^{b} u_{o}^{2}(r) d r
$$

where the prime denotes the differentiate with respect to $r$. Using (3) to evaluate the left hand side in (17), we get

$$
\delta_{0}{ }^{\prime}=2 \sin ^{2} \delta_{o} \int_{0}^{b} \mathrm{u}_{o}^{2}(r) d r+\frac{\sin \left(2 k_{o} b+2 \delta_{o}\right)}{2 k_{o}}-b
$$

Where $\delta_{0}{ }^{\prime}=\frac{\partial \delta_{0}}{\partial k_{0}}$ evaluate at $\mathrm{k}=\mathrm{k}_{0}$.

It is straight forward to show that the last equation may be written as 


$$
\delta_{0}{ }^{\prime}=2\left[\int_{0}^{b} u_{0}^{2}(r) d r-\int_{0}^{b}\left[u_{0}^{e x t}(r)\right]^{2} d r\right] \sin ^{2} \delta_{0}+\frac{\sin 2 \delta_{0}}{2 k_{0}}
$$

where $u_{0}^{\text {ext }}(r)$ is the asymptotic wave function given in (3) evaluated at $\mathrm{k}=\mathrm{k}_{0}$ for all $\mathrm{r}$ values. Substituting the last equation into (15) and rearranging leads to

$$
\alpha_{1}=\frac{2 m}{\hbar^{2}}\left[\int_{0}^{b}\left[u_{0}^{e x t}(r)\right]^{2} d r-\int_{0}^{b} u_{o}^{2}(r) d r\right]
$$

Since for $r \geq R$ the free and interacting wavefunctions are identical, the upper limit of integration (20) may be extended to infinity without changing the result, hence

$$
\alpha_{1}=\frac{2 m}{\hbar^{2}}\left[\int_{0}^{\infty}\left[u_{0}^{e x t}(r)\right]^{2} d r-\int_{0}^{\infty} \mathrm{u}_{o}^{2}(r) d r\right]
$$

When evaluated at zero energy, the above expression gives the Bethe formula for effective range [18].

To find $\alpha_{2}$ we start by noting that (4) and (5) are valid for all values of $k_{0}$. Hence, we may write

$$
u_{n+1}\left(k_{o}, r\right)=\left.\frac{2 m}{\hbar^{2}} \frac{\partial}{\partial k^{2}} u_{n}(k, r)\right|_{k=k_{o}}
$$

and

$$
\alpha_{n+1}=\left.\left(\frac{2 m}{\hbar^{2}} \frac{\partial}{\partial k^{2}}\right)^{n+1} k \cot \delta\right|_{k=k_{o}}=\left.\left(\frac{2 m}{\hbar^{2}}\right) \frac{\partial}{\partial k^{2}} \alpha_{n}\right|_{k=k_{o}}
$$

Furthermore, defining a power series expansion in $\Delta \mathrm{E}$ for $u^{e x t}(k, r)$ similar to that given in (4), then $u_{n+1}^{e x t}\left(k_{o}, r\right)$ can be written as

$$
u_{n+1}^{e x t}\left(k_{o}, r\right)=\left.\frac{2 m}{\hbar^{2}} \frac{\partial}{\partial k^{2}} u_{n}^{e x t}(k, r)\right|_{k=k_{o}}
$$

To find $\alpha_{2}$ we set $\mathrm{n}=1$ into (23) and use (21) to evaluate $\alpha_{1}$ 


$$
\begin{aligned}
\alpha_{2} & =\left.\left(\frac{2 m}{\hbar^{2}}\right) \frac{\partial}{\partial k^{2}} \alpha_{1}\right|_{k=k_{o}} \\
& =\left(\frac{2 m}{\hbar^{2}}\right) \int_{0}^{\infty} 2 u_{0}^{e x t}(r)\left[\frac{2 m}{\hbar^{2}} \frac{\partial}{\partial k^{2}} u_{0}^{e x t}(r)\right] d r-\int_{0}^{\infty} 2 u_{0}(r)\left[\frac{2 m}{\hbar^{2}} \frac{\partial}{\partial k^{2}} u_{0}(r)\right] d r, \quad(25-b)
\end{aligned}
$$

Obviously from (24) the terms in the bracket in the last equation is equal to $u_{1}^{\text {ext }}(r)$ and $u_{1}(r)$ respectively, then we get the final expression for $\alpha_{2}$ namely

$$
\alpha_{2}=\left(\frac{2 m}{\hbar^{2}}\right)\left[\int_{0}^{b} 2 \mathrm{u}_{o}^{e x t}(r) u_{1}^{e x t}(r) d r-\int_{0}^{b} 2 \mathrm{u}_{o}(r) \mathrm{u}_{1}(r) d r\right]
$$

\subsection{Application on the derived formalism}

In his book on scattering theory, Newton [19] showed that there is a finite time delay in the emergence of the scattered particles associated with a change in phase shift. This time delay is expressed as

$$
t_{d}=\left.\frac{2 m}{\hbar k_{0}} \frac{\partial \delta_{l}}{\partial k}\right|_{k=k_{0}}
$$

this equation indicates when $\boldsymbol{\partial} \boldsymbol{\delta} /\left.\boldsymbol{\partial} \boldsymbol{k}\right|_{\boldsymbol{k}=\boldsymbol{k} \boldsymbol{0}}$ is positive, the emerging scattered particles experience time delay, while when negative then an early emergence rather than a delay occurs. For particles whose energies are below the height of a potential barrier and far from a resonance the slope is negative and the scattered particles emerge faster than the case when the barrier is absent. This is in an excellent agreement with what one expects from classical physics. At a resonance the phase shift has a large positive slope leading to a large time delay. In quantum mechanics this corresponds to the fact that some particles tunnel through the barrier region into the interior region and they must again pass through the barrier to escape from the interaction region [1] . Our work is relevant here as the time delay may be expressed in terms of the change 
of the phase shift when the incident energy is changed. Deriving analytic expressions for the change of the phase shifts when the energy is chanced by a small amount using a velocity dependent potential has been the main purpose of this work.

\section{Data and Results}

In this section we shall use the formula of section 2 to calculate the scattering phase shifts in the $\mathrm{S}$-wave case when the energy is changed by a small amount $\Delta \mathrm{E}$ from an arbitrary unperturbed value $\mathrm{E}_{0}$. The validity of our results shall be tested by comparing the perturbed phase shifts to those obtained exactly by solving Schrödinger equation. We considered the local potential to have the form of a finite square well;

$$
\mathrm{V}(\mathrm{r})=-\mathrm{V}_{0} \theta(\mathrm{r}-\mathrm{b})
$$

where the step function is defined as

$$
\theta(\mathrm{r}-\mathrm{b})=1, \quad \mathrm{r}<\mathrm{b} \quad \text { and } \quad \theta(\mathrm{r}-\mathrm{b})=0, \quad \mathrm{r} \geq \mathrm{b}
$$

but the velocity-dependent part is taken to have the form of a Yukawa potential [20];

$$
\rho(r)=\frac{-\rho_{0} \exp (-a r)}{r}
$$

where $\rho_{0}$ and $1 / \mathrm{a}$ are respectively the strength and range of the nuclear force.

Our form for $\mathrm{V}_{0}$ is that adopted by Razavy et al.[6] who modeled the neutron-proton scattering at low energy using a velocity-dependent potential. They managed to reproduce the ${ }^{1} \mathrm{~S}$, ${ }^{1} \mathrm{D}$ and ${ }^{1} \mathrm{G}$ singlet-even phase shifts for the proton-neutron interaction. Therefore, we shall adopt their values for $V_{0}$ and $\mathrm{b}$, namely $V_{0}=16.9 \mathrm{MeV}, \mathrm{b}=2.4 \mathrm{fm}$.

The parameters $\rho_{0}$ and $a$ were chosen such that we recovered, as closely as possible, the exact unperturbed scattering phase shifts $\delta_{0}$ given in Ref. [6]. The parameters are $\rho_{0}=1 \mathrm{MeV}$ and a $=0.5$ fm.

To find the exact perturbed phase shift as a function of the center of mass energy which is perturbed by a small amount $\Delta \mathrm{E}=0.15 \mathrm{E}_{0}$ we solved the Schrödinger equation (equation 2) numerically to 
find the perturbed wave functions in the internal region $r<b$ and the asymptotic region $r>b$. Since $\rho(r)$ is continuous, the wave function and its derivative arecontinuous at $\mathrm{r}=\mathrm{b}$.

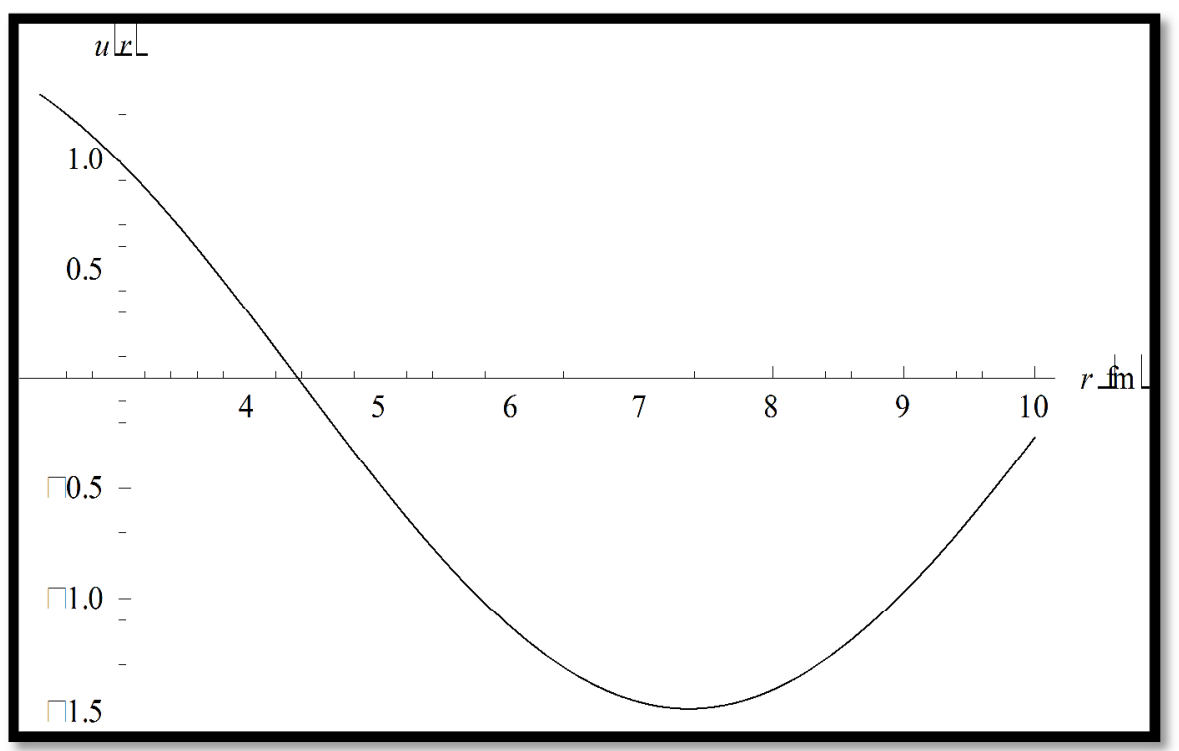

Figure 1: The wave function versus $r$ corresponding to incident energy of $10 \mathrm{MeV}$.

By determining the interception of the external wave function with $r$-axis (for example in Fig.1, for $\mathrm{E}=10 \mathrm{MeV}$ the first interception point occurs at $r_{0}=4.3731 \mathrm{fm}$ ). The exact perturbed phase shift is then calculated by setting [21]

$$
\sin \left(k r_{0}+\delta\right)=\sin k r_{0} \cos \delta+\cos k r_{0} \sin \delta=0
$$

we determined larger values of $\mathrm{r}$ where $u(r)$ intercepts the r-axes and the corresponding values of $\delta$ were the same. Similarly, the exact unperturbed scattering phase shifts $\delta_{0}$ were determined by setting $\Delta \mathrm{E}=0$ and listed in the second column of Table 1 .

In order to determine the perturbed phase shifts $\delta^{\text {per }}$ we calculated $\alpha_{0}, \alpha_{1}$, and $\alpha_{2}$ using (5), (21), and (26) then the perturbed phase shifts values $\delta^{\text {per }}$ determined up to second order as listed in Table 1. At small perturbation the agreement between the perturbed phase shifts $\delta^{\text {per }}$ and the exact phase shifts $\delta$ is expected to be good, this is clearly shown in table 1 for low energy since $\Delta \mathrm{E}$ is small. For example, for $\mathrm{E}$ $=5 \mathrm{MeV}$ the exact phase shift $\delta=1.026$ in good agreement with 1.032 obtained using the perturbation approach. The percentage error $\Delta \delta_{e r r}=\frac{\delta-\delta^{p e r}}{\delta} \times 100 \%=0.58 \%$. 
However, as the energy increases the discrepancy tends to grow larger, this is clearly seen by inspecting the values of $\Delta \delta_{\text {err }}$ in Table 1 for $E=50 \mathrm{MeV}$ and $120 \mathrm{MeV}$. This can be explaining by noting that $k \cot \delta$ in (5) is expanded in a powers of $\Delta \mathrm{E}$. This is because $\Delta \mathrm{E}$ gets larger the higher order terms in the effective range expansion become significant.

\begin{tabular}{|c|c|c|c|c|c|c|c|}
\hline $\begin{array}{c}\mathrm{E}_{0 \mathrm{CM}} \\
(\mathrm{MeV})\end{array}$ & $\delta_{0}(\Delta \mathrm{E}=0)$ & $\delta$ (exact) & $\alpha_{0}$ & $\alpha_{1}$ & $\alpha_{2} \times 10^{-3}$ & $\delta^{p^{\mathrm{er}}}$ & $\Delta \delta_{\text {err }}$ \\
\hline 5 & 1.055 & 1.026 & 0.2227 & 0.0343 & 0.0004 & 1.032 & 0.58 \\
\hline 10 & 0.884 & 0.839 & 0.4041 & 0.0395 & 0.0223 & 0.849 & 1.19 \\
\hline 15 & 0.749 & 0.698 & 0.6456 & 0.0459 & 0.0465 & 0.711 & 1.86 \\
\hline 50 & 0.214 & 0.142 & 5.0459 & 0.2422 & 2.568 & 0.168 & 18.3 \\
\hline 120 & -0.241 & -0.317 & -6.9186 & -0.0146 & -1.088 & -0.243 & 23.34 \\
\hline
\end{tabular}

Table 1.The third column gives the exact ${ }^{1} \mathrm{~S}$ perturbed phase shifts $\delta$ as a function of the center of mass energy which is perturbed by $\Delta \mathrm{E}=0.15 \mathrm{E}_{0}$. The parameters of the effective range expansions are shown in columns $4-6$. Column 7 gives the perturbed phase shifts $\delta^{p e r}$ calculated up to second order. 
Also, we used the same potential forms and determined the perturbed phase shifts corresponding to a constant small energy perturbation $\Delta \mathrm{E}=0.75 \mathrm{MeV}$ and the results are shown in Table 2. As expected since the perturbation is small the agreement between $\delta^{p \text { er }}$ and $\delta$ are quite good for all energies.

\begin{tabular}{|c|c|c|c|c|c|c|c|}
\hline $\begin{array}{c}\mathrm{E}_{0 \mathrm{CM}} \\
(\mathrm{MeV})\end{array}$ & $\delta_{0}(\Delta \mathrm{E}=0)$ & $\delta$ (exact) & $\alpha_{0}$ & $\alpha_{1}$ & $\alpha_{2} \times 10^{-3}$ & $\delta p^{\mathrm{er}}$ & $\Delta \delta_{\text {err }}$ \\
\hline 5 & 1.055 & 1.025 & 0.1968 & 0.0343 & 0.0004 & 1.032 & 0.58 \\
\hline 10 & 0.884 & 0.861 & 0.4025 & 0.0394 & 0.0222 & 0.867 & 0.69 \\
\hline 15 & 0.749 & 0.732 & 0.6456 & 0.0459 & 0.0469 & 0.736 & 0.55 \\
\hline 50 & 0.214 & 0.206 & 5.0459 & 0.2423 & 2.5689 & 0.208 & 0.97 \\
\hline 120 & -0.241 & -0.244 & -6.9186 & -0.0147 & -1.0883 & -0.242 & 0.82 \\
\hline
\end{tabular}

Table 2.The third column gives the exact ${ }^{1} \mathrm{~S}$ perturbed phase shifts $\delta$ as a function of the center of mass energy which is perturbed byconstant energy perturbation $\Delta \mathrm{E}=0.75 \mathrm{MeV}$. The parameters of the effective range expansions are shown in columns 4-6. Column 7 gives the perturbed phase shifts $\delta^{p e r}$ calculated up to second order.

Figure 2 shows the unperturbed wavefunction $\mathrm{u}_{0}(\mathrm{r})$ and the corresponding first-order correction $\mathrm{u}_{1}(\mathrm{r})$ correspond to $\mathrm{E}=120 \mathrm{MeV}$. Clearly, the amplitude of the wavefunction correction $\mathrm{u}_{1}(\mathrm{r})$ is smaller than the amplitude of the unperturbed wavefunction $\mathrm{u}_{0}(\mathrm{r})$. This reflects the progressive decrease in the contribution to the perturbed phase shifts arising from higher order correction. 


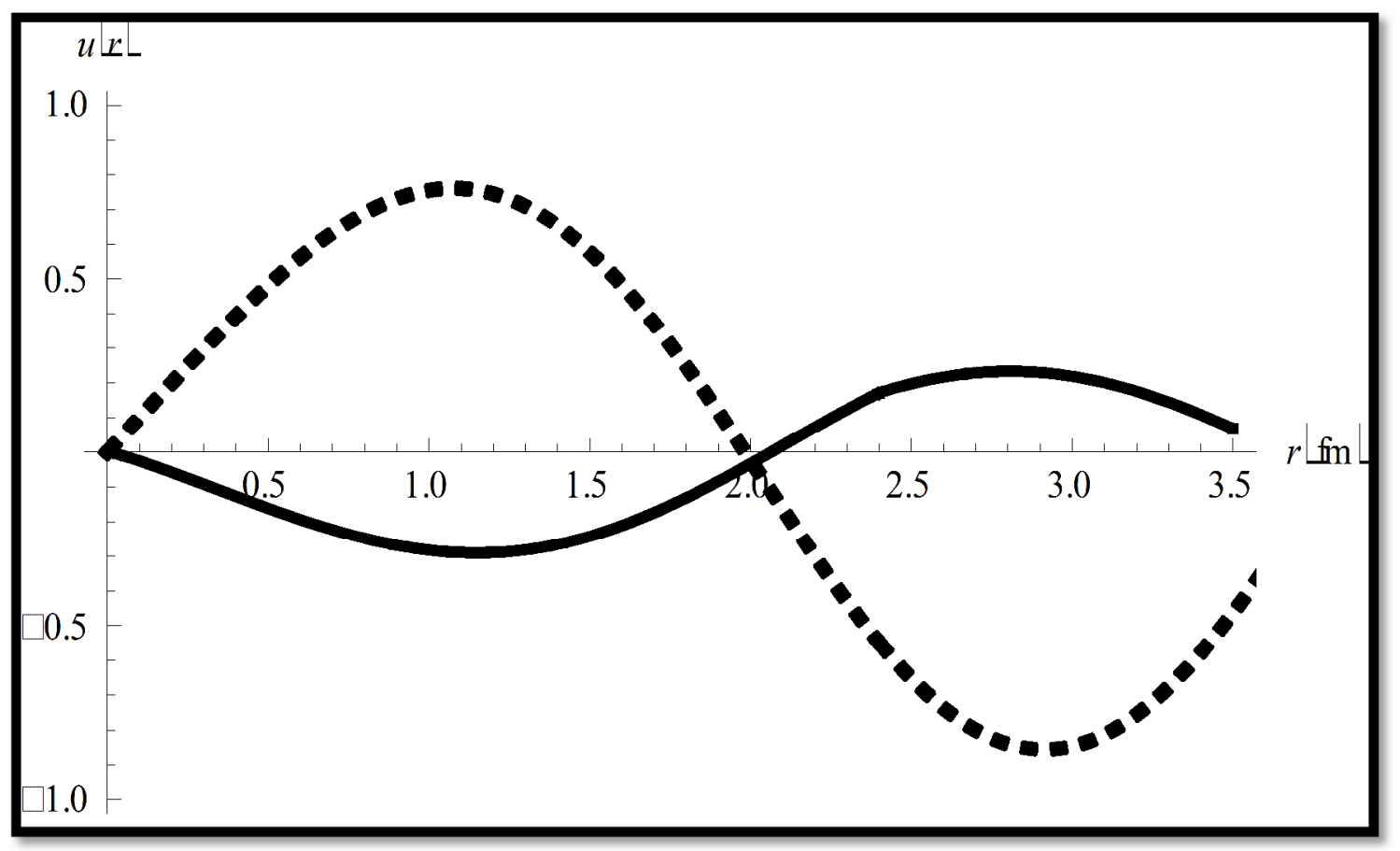

Figure 2: The unperturbed wavefunction $\mathrm{u}_{0}$ (dotted line), the first-order wavefunction correction $\mathrm{u}_{1}$ (solid line) correspond to $\mathrm{E}=120 \mathrm{MeV}$.

\section{Conclusions}

In this work we considered the time independent Schrödinger equation in the presence of velocity dependent potential. We have determined the scattering phase shifts in the S-wave case when the incident energy is perturbed by a small amount $\Delta \mathrm{E}$ from its original value $\mathrm{E}_{0}$. We assumed the local and velocity-dependent parts of the potential to have the form of a finite square well and a Yukawa potential respectively. The exact phase shifts as a function of incident energy were obtained numerically and the result are shown in the third column of Table 1 corresponding to a small perturbing energy $\Delta E=0.15 \mathrm{E}_{0}$. We calculated $\alpha_{0}, \alpha_{1}$ and $\alpha_{2}$ and hence determined the perturbed phase shifts $\delta^{\text {per }}$ up to second order using (5) and the results are shown in the last column of Table 1.

As shown in Table 1when the perturbing energy is small the agreement between the exact and the perturbed phase shifts is good. However, as the energy increases, $\Delta \mathrm{E}$ grows larger so the results becomes progressively worse. We have calculated the values of perturbed phase shifts corresponding to a constant perturbing energy $\Delta \mathrm{E}=0.75 \mathrm{MeV}$ and the results are shown in Table 2. The agreement between the perturbed phase shifts $\delta^{\text {per }}$ and the corresponding exact ones $\delta$ is 
perfectly good for all energies since $\Delta \mathrm{E}$ is small. For example, for $\mathrm{E}=10 \mathrm{MeV}$ we found $\delta^{\text {per }}=$ 0.867 in good agreement with the exact result of 0.861 .

\section{Acknowledgment}

The financial support from university of Tabuk is gratefully acknowledged.

\section{References}

[1] Romo, W. J., Valluri, S. R: Nucl. Phys.A 636, 467 (1998).

[2]Milward, G. and Wilkin, C.: J. Phys. A: Math.Gen. 34, 5101 (2001).

[3] Blatt, J. M., Jackson, J. D.: Phys. Rev. 26, 18 (1949).

[4] Lodhi,M.A.K.: Phys. Rev. 182, 1061 (1969).

[5] Green, A.E. S.,Darewych, G.,Berezdivin, R.: Phys. Rev. 157, 929 (1967).

[6] Razavy, M., Field, G.,Levinger, J. S.: Phys. Rev. 125, 269 (1962).

[7] Razavy, M.:Nucl. Phys. 50, 465 (1964).

[8] Green,A.M.:Nucl. Phys. 33, 218 (1962).

[9] LIN, H.E., LIN, W.C.: Nuc. Phys. B 16, 431 (1970).

[10]Bhaduri, R.K.,Preston,M.A.: Can. J. Phys. 42, 696 (1964).

[11] Green, A.E.S.,Sharma,R.D.: Phys. Rev. Lett. 14, 380 (1965).

[12] Miller,L.D. ,Green,A. E. S.: Phys. Rev. 184, 1012(1969).

[13] Bayrak, O., Soylu, A., Boztosun, I: Cent. Eur. J. Phys. 10, 953 ( 2012).

[14] Kisslinger,L. S.: Phys. Rev. 98, 761 (1955).

[15] Appel, K., Werner, E.: Nucl. Phys. 38, 74 (1962).

[16] Schiff, L. I.: Quantum mechanics, New York, McGraw-Hill (1968).

[17] Al-Sayyed, M. , Jaghoub, M.I.: Int J Theor Phys. 50, 3357 (2011). 
[18] Bethe, H. A., Phys. Rev. 76, 38 (1949).

[19] Newton, R.G.: Scattering Theory of Waves and Particles (Springer, New York, 1982).

[20] Sakurai, J.J.: Modern Quantum Mechanics, Addison-Wesley publishing company, Inc., New York (1994).

[21] Bertulani, C. A.: Nuclear physics in a nutshell, Princeton university press (2005). 